\title{
Surface Texture Detection with Artificial Fingers*
}

\author{
N.H.H. Mohamad Hanif, Paul H. Chappell, Andy Cranny, Neil M. White
}

\begin{abstract}
This paper highlights the potential of using prosthetic devices to sense surface textures; an important characteristic of a lower arm that is often neglected. An artificial finger equipped with a piezoelectric sensor, mounted on a fingertip, has been designed to detect surface textures of different dimensions. Signal frequencies generated during the exploratory movement of the artificial finger reliably correlate to all the widths of grooves and ridges of the surface textures under investigation. This capability provides a positive outlook in recreating a touch sensation that has been previously lost from natural fingers and palms.
\end{abstract}

\section{INTRODUCTION}

According to the Office for Disability Issues Estimates 2012, there are 11.6 million disabled people living in the United Kingdom, in which 6.3 million of them have an impairment of lifting and carrying [1]. To assist in daily living, the use of prosthetic devices have become a norm and generally well accepted in society. For simple cosmetic purposes and pick-and-place functions, prosthetic devices have evolved into sophisticated devices, to closely mimic the ability of natural hands. While there are many commercial prosthetic devices in the market, very little of them are equipped with surface texture sensation capabilities, which would have provided a more natural feeling to the users. The absence of a sensing modality creates difficulties for users to control a device, as there is insufficient information about the object they are manipulating [2].

Realizing the importance in restoring the sense of touch for amputees, there has been international research in this field. In research by Song, different surface properties of various objects were classified using a three-fingered robotic hand equipped with force-torque sensor [3]. Research by $\mathrm{Su}$ incorporates artificial fingertips with liquid biomimetic tactile sensors to discriminate different varieties of rubber samples [4]. Recently a $£ 1.4$ million fund has been allocated by the Engineering and Physical Sciences Research Council (ESPRC) for a project on bionic hands that not only provides information about the hand's position and movement, but also is sensitive to pressure and temperature [5].

Our research works uses piezoelectric sensors to decipher surface gratings in terms of the widths of grooves and ridges. The widths of the ridges and grooves of the gratings in one spatial period play an important role in perceived roughness. As reported in [6], wider groove width leads to better

*Research supported by Ministry of Education, Malaysia.

N.H.H. Mohamad Hanif is with the Electronics \& Computer Science, University of Southampton, UK. (Phone: +44(0)2380594996; fax: +44(0)2380592901; e-mail: noorhazrin@iium.edu.my), on study leave from the International Islamic University Malaysia.

Paul H. Chappell, Andy Cranny and Neil M. White are with the Electronics \& Computer Science, University of Southampton, UK. (Phone: $+44(0) 238059$ 3442; fax: $+44(0) 23 \quad 8059$ 2901; email: phc@ecs.soton.ac.uk) estimation of roughness perception while wider ridges results in lower estimation of perceived roughness. This published psychophysics investigation will be compared with the sensation acquired by the piezoelectric sensor from the Southampton artificial finger.

\section{DATA ACQUiSITION STRATEGIES}

\section{A. Test Apparatus}

A previously built test assembly by Muridan [7] has been used and modified for this research work. The test rig consists of a hollow aluminum sliding tube that is driven by a DC motor and toothed drive belt. A linear, digital encoder measures the position of the sliding tube relative to an aluminum base. The velocity of the block can be varied by varying the voltage supply to the DC motor. A finger link that holds the piezoelectric sensor is positioned at the middle of the sliding block. The information about the velocities and the output voltage from the sensor were recorded using a high resolution $200 \mathrm{kSs}^{-1}$ data acquisition device by National Instruments (NIDAQPad 6016). The test apparatus is as shown in Figure 1.

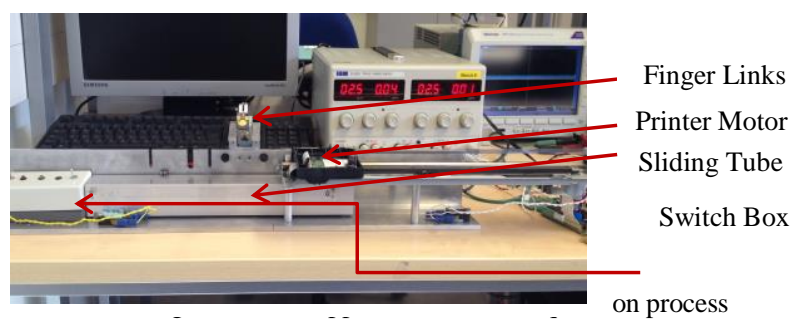

B. Piezoelectric Sensor

A piezoelectric sensor has been used to acquire signals when it was slid against textured surface. This sensor is designed to mimic the human mechanoreceptors at the fingertips. The sensor is mounted at the end of the finger link and held secure with small bolts. The sensor used for this work has been developed by Cranny [8] and Cotton [9]. Figure 2 illustrates the sensor with its finger link system.

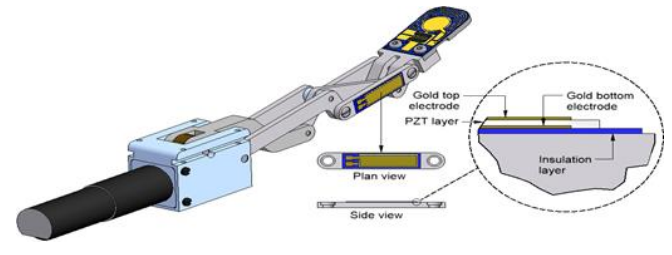

Figure 2: The finger link system (illustration credit to Dr. Andy Cranny, University of Southampton)

Referring to Figure 2, the yellow circular shape is the dynamic force sensor. This is the area of contact between the sensor and the textured surface. This part of sensor is made of 
four layers which are insulation layer, gold bottom electrode, PZT layer and gold top electrode. The positive and negative electrical charges generated by the PZT layer due to the contact with the surface will move to the gold top electrode and gold bottom electrode respectively. The potential difference will be amplified with a suitable voltage amplifier and a voltage waveform will be produced and could be observed with an oscilloscope (Figure 3).

To conduct the experiment, the finger link is mounted on a bracket. This will put the finger in a stationary position while the block with the textured surface slides along the aluminium base.

\section{Design of Voltage Mode Amplifier}

A voltage amplifier has been carefully designed to interface with the sensor. This amplifier is used to produce voltage output detected by the sensor within the required frequency range and signal amplitude. An instrumentation amplifier has been chosen due to its high input impedance and to produce less distortion to the original signal. This characteristic is important for this design as the piezoelectric sensor has large input impedance, and to minimize the loading effect, comparable input impedance is required [10]. The schematic design of the voltage mode amplifier is as shown in Figure 3.

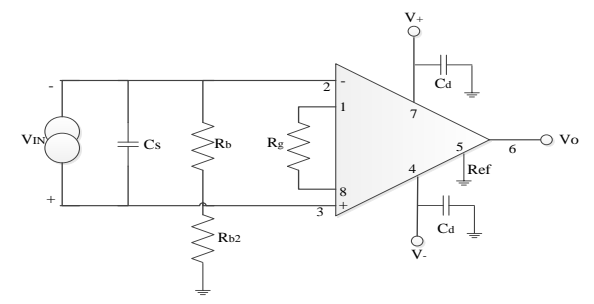

Figure 3: Schematic design of a voltage mode amplifier

\section{Encoder}

The two position signals from the linear encoder were also acquired in order to measure the velocity of the sliding block.

The encoder of the experiment rig consists of a series of black and white stripes (Figure 4). By determining the width of a pair of black and white stripes, the encoder velocity can be calculated. The widths were measured by gathering the data samples during the sliding motion of the block. These samples were acquired using the NIDAQPad-6016. Five measurements were taken and the mean of the width was calculated. The width of one pair of the encoder stripe has been determined to be $0.0865 \mathrm{~mm}$.

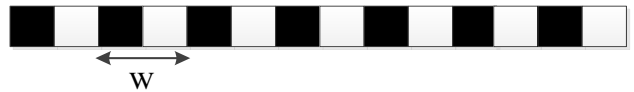

Figure 4: Linear encoder of the rig. W, the width of a pair, is $0.0865 \mathrm{~mm}$.

\section{DATA ACQUisition OF SURFACE TEXTURES}

\section{A. Surface Textures}

Four surface textures with different dimensions were investigated. Test on a smooth surface (Type A) was conducted for control purposes. Type B texture has repeating patterns with similar widths of grooves and ridges. Type $\mathrm{C}$ texture consists of repeating patterns of different widths of ridges and constant widths of grooves. Type $\mathrm{D}$ texture has a set of repeating patterns of increasing widths of grooves and constant widths of ridges. Each grating textures were made with $310 \mathrm{~mm}$ acrylic strips. The length per texture is 100 $\mathrm{mm}$. Figure 5 highlights the groove, ridge and spatial period in gratings while Figure 6 shows the dimensions of the surface textures under investigation.

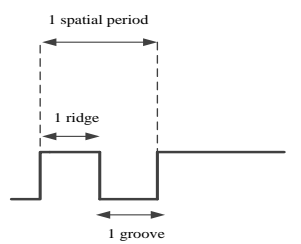

Figure 5: Physical parameters of a grating

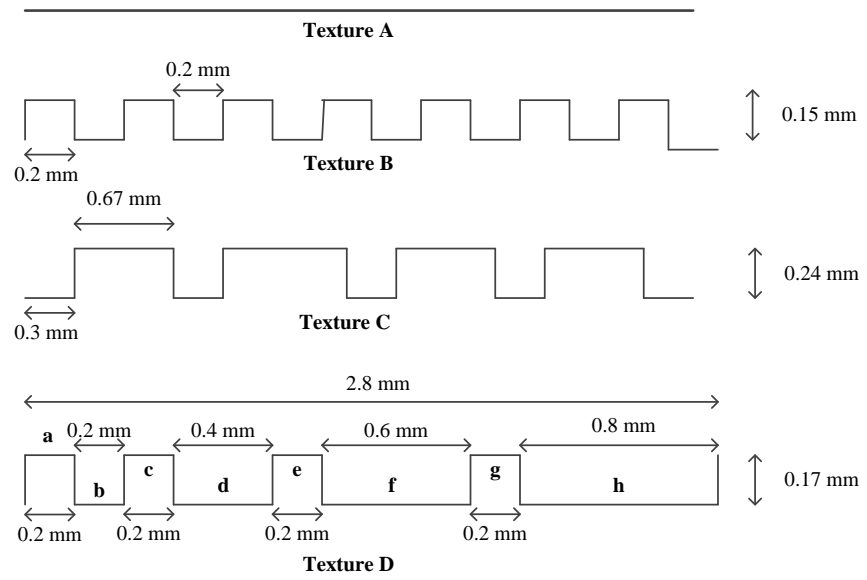

Figure 6: Dimensions of Surface Textures under Investigation (Texture A: smooth surface, Texture B: repeating every $0.4 \mathrm{~mm}$, Texture C: repeating every $0.97 \mathrm{~mm}$, Texture D: repeating every $2.8 \mathrm{~mm}$ )

\section{B. Experiments}

The acrylic strip that consists of the fabricated surface texture was attached to the sliding block using screws at both ends of the strip. By having these removable strips, investigations on different types of surface texture could be conducted as these strips could be easily removed and reattached. The piezoelectric sensor, mounted on the fingertip and layered with a silicone cosmesis glove, was positioned on the vertical side of the surface texture. The contact force between the sensor and the surface was $60 \mathrm{mN}$, which was approximately the mass of the fingertip. This value was not measured or controlled, but to ensure that the sensor could detect reliable signals, there should be a very minimal gap between both surfaces. On the other hand, the contact force should not be very high to avoid unnecessary friction that may in the extreme, result in the block not sliding. Figure 7 shows the top view illustration of the moving block and the piezoelectric sensor. 


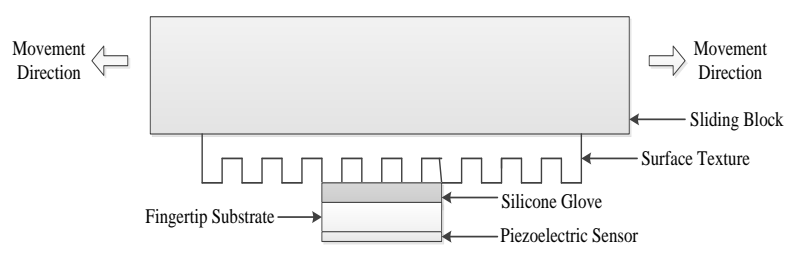

Figure 7: Top view illustration of the moving block and the piezoelectric sensor

\section{OUTPUT SIGNALS}

\section{A. Texture A (Smooth Surface)}

This texture is a part of the control data, in which the purpose was to identify existing signals / noise so that it would not be mistaken as signals generated by the fabricated surface gratings. At a constant velocity of $77.1823 \mathrm{~mm} / \mathrm{s}$, a noise component frequency magnitude was observed at 4.589 $\mathrm{Hz}$.

\section{B. Texture B (Similar widths of grooves and ridges)}

For this surface texture, velocities were varied between 20 $\mathrm{mm} / \mathrm{s}$ to $135 \mathrm{~mm} / \mathrm{s}$. At different velocities, components of the signals from an FFT analysis corresponded to the widths of the surface gratings. The clearest outputs however, were achieved when the velocity of the block moved at more than $90 \mathrm{~mm} / \mathrm{s}$. At these velocities, the FFT components were the dominant frequencies. Figure 8 shows the encoder and piezoelectric signals obtained at velocity of $95.6 \mathrm{~mm} / \mathrm{s}$.
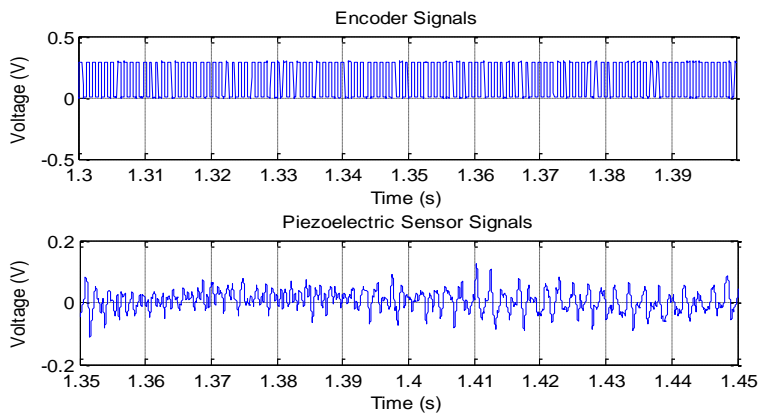

Figure 8: Texture B - Encoder and Piezoelectric Signals at $95.6 \mathrm{~mm} / \mathrm{s}$

A dominant frequency of $468.1 \mathrm{~Hz}$ was detected for the selected sample length, as shown in Figure 9. From this frequency and the measured velocity, the widths, $w$ of the ridge/groove could be calculated by dividing the frequency, $\mathrm{f}$, with the velocity, $\mathrm{v}$, which is equivalent to $0.2042 \mathrm{~mm}$. The accurate measurements from microscope measurements are $0.2001 \mathrm{~mm}$ for the ridge and $0.19258 \mathrm{~mm}$ for the groove. These are $2.0 \%$ and $-3.76 \%$ differences between the calculated and measured widths of ridge and groove respectively.

It could be observed that there is only one dominant FFT output for this particular texture. This is because the texture has a repeating groove and ridge at similar widths.

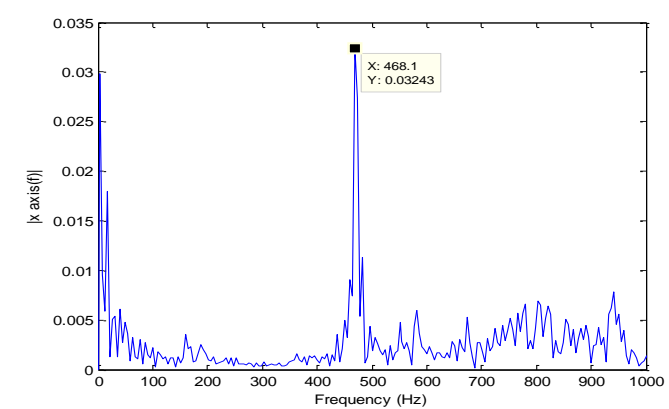

Figure 9: Texture B -FFT Output

\section{Texture C (Different widths of grooves and ridges)}

Data were gathered when the sliding block was moving between $80-140 \mathrm{~mm} / \mathrm{s}$. As texture C consists of two different widths, the FFT output shows two dominant frequencies corresponding to the widths. The plot also shows occurrence of a third frequency at a lower magnitude, which is the sum of the two dominant frequencies. The following plots show the signals generated when velocity was constant at $136.3 \mathrm{~mm} / \mathrm{s}$.
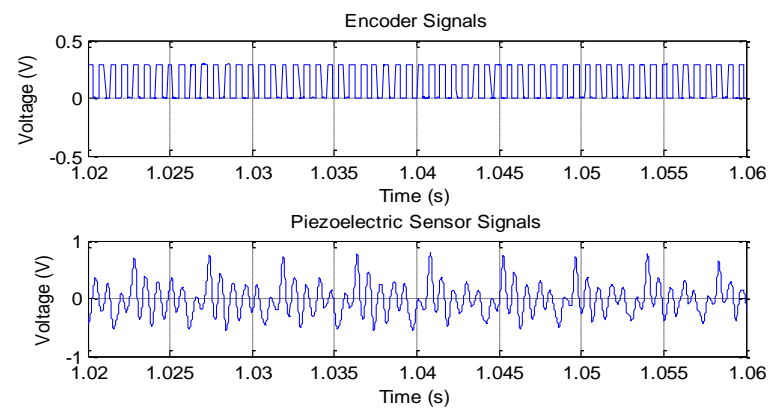

Figure 10: Texture C - Encoder and Piezoelectric Signals at $136.3 \mathrm{~mm} / \mathrm{s}$

At this velocity, the calculated width of a groove is $0.2803 \mathrm{~mm}$, which is $7.1 \%$ different than the measured groove width. The calculated width of ridge is $0.5606 \mathrm{~mm}$, that is a $20.5 \%$ difference with the measured value. The frequencies associated with the width of the grooves are more dominant than the frequency associated with the width of ridges.

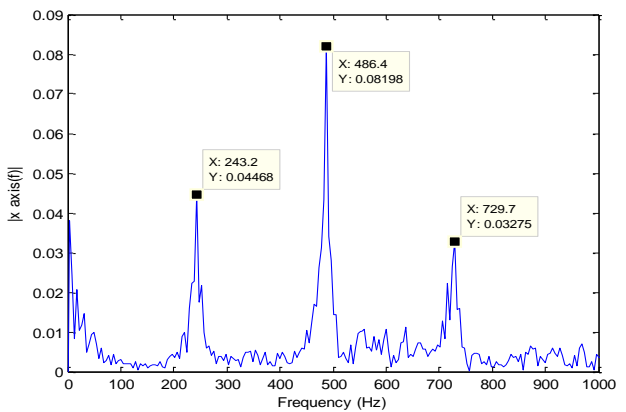

Figure 11: Texture $C$-FFT Output

\section{Texture D (Increasing widths of grooves and constant widths ridges)}

Experiments conducted at varying constant velocities have shown the capability of the sensor to produce output representing the surface textures. At velocity of 
$139.35 \mathrm{~mm} / \mathrm{s}$, dominant frequencies were detected at 183.6 $\mathrm{Hz}$, which corresponds to $0.7099 \mathrm{~mm}$ groove width $(8.68 \%$ difference with the measured groove width), $215.7 \mathrm{~Hz}$ which corresponds to $0.604 \mathrm{~mm}(-1.63 \%$ different than the measured groove width), $275.3 \mathrm{~Hz}$ which corresponds to $0.473 \mathrm{~mm}(-14.88 \%$ difference than with the measured groove) as well as $642.5 \mathrm{~Hz}$ which corresponds to $0.203 \mathrm{~mm}$ (3.4\% difference than the measured groove width). Figure 12 highlights the FFT output generated for Texture D.

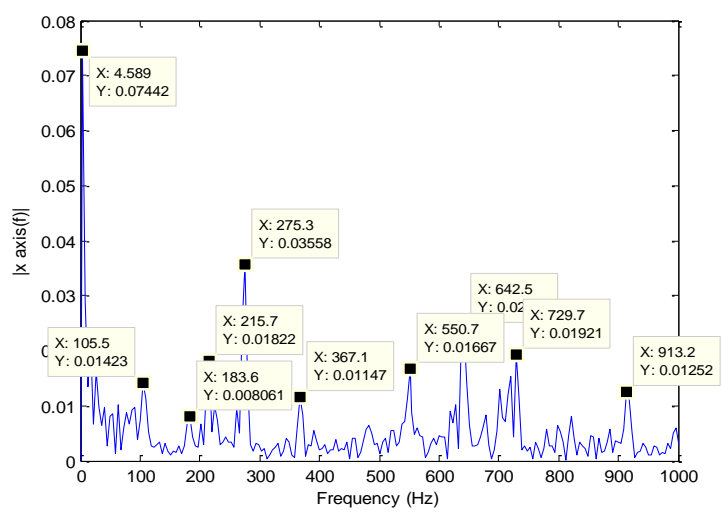

Figure 12: Texture D-FFT Output

\section{DISCUSSIONS}

This research work has shown a significant improvement in sensing surface textures. At velocities ranging from very low to very high, the widths of every ridge and groove were repeatedly and successfully identified. In previous work[7], the capability of the artificial finger was just limited to detecting the combination width of a ridge and a groove of the surface. By modifying the amplifier circuit configuration, the sensitivity of the piezoelectric sensor has improved tremendously.

The existence of a low frequency component $(4.589 \mathrm{~Hz})$ in every surface, including the smooth surface, is possibly due to external factors. The cause of a low frequency 'noise' could be many and varied, such as a low hum generated by plant in the infrastructure of a building. In all findings, it has been observed that the frequencies corresponding to the groove widths are more dominant (higher) than the frequencies corresponding to the widths of ridges. This implies that the piezoelectric sensor is most sensitive in detecting grooves than in detecting ridges. This observation matches with the capability of human mechanoreceptors at the fingertips that could perceive roughness much better at a groove of a surface rather than at the ridge [6]. Psychophysics investigation by Lederman [11] showed that as the widths of a ridge is increased, the estimation of roughness becomes lower. This characteristic is observed with Texture $\mathrm{C}$ in which the percentage difference between the actual and measured width is larger as compared to other surface textures with narrower ridge widths.

Although the grating widths could be detected at different ranges of velocity, the artificial finger is at its best sensitivity when the velocity was between $90 \mathrm{~mm} / \mathrm{s}$ to $130 \mathrm{~mm} / \mathrm{s}$. This is when the generated FFT frequencies corresponding to the grating widths are the dominant frequencies. This finding is similar as with the previous work [7] in which dominant frequencies associated to the grating widths were observed at velocities above $90 \mathrm{~mm} / \mathrm{s}$. This is most probably the optimum velocity range for the piezoelectric sensor, which is within the capability range of a human finger. According to Taylor, velocity has a very minimal effect on perceived roughness of a surface [12]. A person can still discriminate surface texture at a speed of up to $207.3 \mathrm{~mm} / \mathrm{s}$ for grating widths greater than $500 \mu \mathrm{m}[13]$.

\section{CONCLUSION AND FUTURE RESEARCH}

The capability of the artificial fingertip to accurately determine the widths of every groove and ridge of a surface texture at different velocities is a stepping-stone in providing supplementary sensations to the human residual arm. The information gathered from the piezoelectric sensor could be translated to vibration to be felt at the upper arm, which will be the next stage of this research work. The test rig shall also be further modified to achieve a constant sliding speed throughout the acquisition process. This ultimately will reduce the processing time as the task for selecting constant velocity is eliminated.

\section{REFERENCES}

[1] “Disability Prevalence Estimates 2011 / 12," 2014.

[2] R. S. Dahiya, G. Metta, M. Valle, and G. Sandini, "Tactile SensingFrom Humans to Humanoids," IEEE Trans. Robot., vol. 26, no. 1, pp. 1-20, Feb. 2010.

[3] X. Song, H. Liu, J. Bimbo, K. Althoefer, and L. D. Seneviratne, "Object Surface Classificaiton based on Friction Properties for Intelligent Robotic Hands," in World Automation Congress, 2013, pp. $1-5$.

[4] Z. Su, J. a Fishel, T. Yamamoto, and G. E. Loeb, "Use of tactile feedback to control exploratory movements to characterize object compliance.," Front. Neurorobot., vol. 6, no. July, p. 7, Jan. 2012.

[5] "Bionic hand that is 'sensitive' to touch and temperature," Newcastle University, 24-Feb-2015.

[6] M. A. Lawrence, R. Kitada, R. L. Klatzky, and S. J. Lederman, "Haptic roughness perception of linear gratings via bare finger or rigid probe," Perception, vol. 36, no. 4, pp. 547-557, 2007.

[7] N. Muridan, "Sensing Texture Using An Artificial Finger," University of Southampton, 2013.

[8] A. Cranny, D. P. J. Cotton, P. H. Chappell, S. P. Beeby, and N. M. White, "Thick-film force, slip and temperature sensors for a prosthetic hand," Meas. Sci. Technol., vol. 16, no. 4, pp. 931-941, Apr. 2005.

[9] D. Cotton, P. Chappell, and A. Cranny, "A novel thick-film piezoelectric slip sensor for a prosthetic hand," IEEE Sens. J., vol. 7, no. 5, pp. 752-761, 2007.

[10] I. Altinay and A. Muralidhar, "Phoenix Ambulatory Blood Pressure Monitor Project," 2007.

[11] S. J. Lederman and M. M. Taylor, "Fingertip force,surface geometry and the perception of roughness by active touch," Percept. Psychophys., vol. 12, no. 1972, pp. 401-408.

[12] M. M. Taylor and S. J. Lederman, "Tactile Roughness of grooved surfaces: A model and the effect of friction," Percept. Psychophys., vol. 17 , no. 1, pp. 23-36, 1975.

[13] S. J. Lederman, R. L. Klatzky, C. L. Hamilton, and G. I. Ramsay, "Perceiving roughness via a rigid probe: Psychophysical effects of exploration speed and mode of touch," Haptics-e Electron. J. Haptic Res., vol. 1, no. 1999, pp. 1-21. 